\title{
Glioblastoma-secreted soluble CD44 activates tau pathology in the brain
}

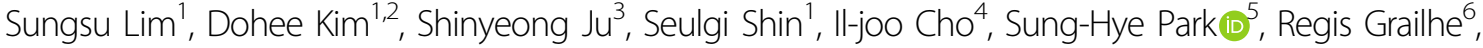 \\ Cheolju Lee ${ }^{3}$ and Yun Kyung Kim ${ }^{1,7}$
}

\begin{abstract}
During aggressive tumor growth and migration, glioblastoma cells secrete diverse molecules and adhesion proteins to the extracellular matrix. Yet, the biochemical effects of the glioblastoma secretome in the brain remain largely unknown. Here we show that soluble CD44 secreted from glioblastoma cells induces neuronal degeneration through the activation of tau pathology in the brain. Glioblastoma-xenograft tissues showed a number of degenerating neurons bearing highly phosphorylated tau. Through a series of secretome-analyses, we identified that soluble CD44 was the responsible protein inducing tau phosphorylation and aggregation $\left(E C_{50}=19.1 \mathrm{ng} / \mathrm{mL}\right.$ ). The treatment of sCD44 to primary hippocampal neurons-induced tau hyperphosphorylation, leading to neuronal degeneration. Also, the injection of sCD44 into the brains of tau transgenic mice induced tau hyper-phosphorylation in hippocampal neurons. Altogether, our data suggest a neurodegenerative role of sCD44 in promoting tau pathology and serving as a molecular link between glioblastoma and neurodegeneration.
\end{abstract}

\section{Introduction}

Glioma is a collection of tumors arising from glial cells, accounting for $>70 \%$ of primary brain tumors ${ }^{1,2}$. The most aggressive type of glioma is clinically classified as glioblastoma ${ }^{3}$. Distinct from other brain tumors, glioblastoma cells have a remarkable tendency to infiltrate the surrounding brain tissue, especially in the form of a dense synaptic network ${ }^{4}$. Glioblastoma cell infiltration is known to cause severed exons or edema leading to synaptic and neuronal degeneration ${ }^{5-8}$. As a result, triggered neuronal degeneration leads to neurologic deficits in vision, memory, communication, or motor functions ${ }^{5-7}$. However, glioblastoma-mediated neurodegeneration has not been

Correspondence: Cheolju Lee (clee270@kist.re.kr) or Yun

Kyung Kim (yunkyungkim@kist.re.kr)

${ }^{1}$ Korea Institute of Science and Technology (KIST), Brain Science Institute,

Convergence Research Center for Diagnosis, Treatment and Care System of

Dementia, Seoul 02791, Republic of Korea

2Department of Biotechnology, Translational Research Center for Protein

Function Control, College of Life Science and Biotechnology, Yonsei University, Seoul 03722, Republic of Korea

Full list of author information is available at the end of the article

These authors contributed equally: Sungsu Lim, Dohee Kim. seriously investigated in the field of brain injury acquired by radiation- and chemotherapy.

In rapid tumor growth and brain infiltration, glioblastoma cells secrete diverse signaling molecules and adhesion proteins to the extracellular matrix, in order to communicate with the surrounding brain tissue ${ }^{9,10}$. To identify the molecular crosstalk between glioblastoma cells and stromal cells, glioblastoma secretome-analysis has been conducted. In 2011, Sirdeshmukh's group first reported 148 non-redundant glioblastoma-secretome markers ${ }^{11}$. In their analysis, most of the secreted proteins were identified to be proteases, protease inhibitors, and adhesion molecules. Another group led by Yetrib reported the secretomes of four glioblastoma cell lines and identified chitinase-3-like1 (Ch3L1) responsible for glioblastoma invasion ${ }^{12}$. More recently, Lee's research group reported that interleukin-1 plays a critical role in tumor progression by evoking an inflammatory response in the brain $^{13}$. These previous approaches, however, focused on investigating the role of glioblastoma-secretome in the general context of a tumor, not in the context of a brain 
that consists of billions of neurons. In this study, we focused on the identification of glioblastoma-secretome that induces neuronal degeneration.

Tau pathology, given its hypothetical link between glioblastoma and neurons, was examined in this study. Tau is a microtubule binding protein that stabilizes microtubules. When abnormally hyper-phosphorylated, tau dissociates from the microtubule and become insoluble aggregates upon neuronal degeneration ${ }^{14}$. Abnormal tau phosphorylation and aggregation are pathological characteristics of multiple neurodegenerative disorders, including Alzheimer's disease, progressive supranuclear palsy, and corticobasal degeneration ${ }^{15}$. Considering that glioblastoma causes diverse metabolic and mechanical stresses, which are considered as potential causes of tau neuropathology ${ }^{16-18}$, it is essential to examine the association of tau pathology in glioblastoma. In this study, we demonstrated that glioblastoma-secretome activates tau pathology, and identified the responsible protein as glioblastoma-secreted sCD44.

\section{Materials and methods}

Glioblastoma cell culture and collection of conditioned media

Glioblastoma cells (A172, HS683, U87, U373, and T98G) were purchased from ATCC (Manassas, VA). Glioblastoma cells were grown in Dulbecco's modified eagle medium (DMEM) containing 10\% fetal bovine serum (FBS), 100 units $/ \mathrm{mL}$ penicillin, and $100 \mu \mathrm{g} / \mathrm{mL}$ streptomycin at $37^{\circ} \mathrm{C}$ in a humidified atmosphere containing $5 \% \mathrm{CO}_{2}$. To collect glioblastoma-conditioned media (C.M.), glioblastoma cells were washed twice with PBS and cultured in serum-free DMEM for $36 \mathrm{~h}$. Glioblastoma conditioned media were collected and centrifuged at 2000 r.p.m. for $10 \mathrm{~min}$ to remove cell debris.

\section{Exposure of Tau-BiFC expressing cells to glioblastoma C.M}

HEK293 tau-BiFC cells were maintained in DMEM containing 10\% FBS, $100 \mathrm{units} / \mathrm{ml}$ penicillin, $100 \mu \mathrm{g} / \mathrm{mL}$ streptomycin, and $100 \mu \mathrm{g} / \mathrm{mL}$ Geneticin (G418, Sigma) at $37^{\circ} \mathrm{C}$ in a humidified atmosphere containing $5 \% \mathrm{CO}_{2}{ }^{19}$. For the treatment of glioblastoma-conditioned media, HEK293 tau-BiFC cells were plated in $\mu$-clear 96-well plates. HEK293 cells expressing GFP were used as a control. After $12 \mathrm{~h}$ of cell attachment, $25 \%$ of the culture medium was replaced with equal volume of glioblastomaC.M. or HEK293-C.M. At various time points after the treatment, the entire plates were automatically imaged by using Operetta (PerkinElmer) and analyzed using a Harmony 3.1 software (PerkinElmer). All experiments were performed in triplicate. The means and standard deviations (SD) of tau-BiFC intensities were plotted by using a Prism software (GraphPad).

\section{Primary neuronal cell culture and treatment with sCD44}

Primary hippocampal neurons were isolated from day 18 embryonic Sprague-Dawley rat brains as described previously ${ }^{20}$. The neurons were seeded at a density of $1.5 \times 10^{4}$ cells/well on a poly-D-lysine-coated 96 -well plate and maintained in the neurobasal medium at $37^{\circ} \mathrm{C}$ in a humidified atmosphere of $5 \% \mathrm{CO}_{2}$. The neurobasal medium contains $2 \%$ of B27 supplement, $0.5 \mathrm{mM}$ of glutamax, 100 units $/ \mathrm{mL}$ of penicillin, and $100 \mu \mathrm{g} / \mathrm{mL}$ of streptomycin. Every 3 days, $50 \%$ of the medium was replaced with the fresh neurobasal medium.

Purified sCD44 was purchased from Sino Biological Inc and dissolved in PBS. At 7 days in vitro, primary neurons were treated with sCD44 $(120 \mathrm{ng} / \mathrm{mL})$. After $48 \mathrm{~h}$ of $\mathrm{sCD} 44$ treatment, the primary neurons were stained with $\mathrm{NeuO}$, a neuron selective probe ${ }^{21}$, and imaged by using Operetta (PerkinElmer). To analyze neuronal degeneration, neurite lengths were quantified by using the Harmony 3.1 software (PerkinElmer). Error bars indicate the standard deviation of three independent experiments, each performed in triplicate. The statistical analysis was performed via paired $t$-test in Excel and the significance was considered at a level of $p<0.05$.

\section{Immunoblot analysis}

For the immunoblot assay, HEK293 tau-BiFC cells were incubated with glioblastoma-conditioned media or Okadaic acid $(30 \mathrm{nM})$ for $24 \mathrm{~h}$. Cell lysates were prepared by using CelLyticM lysis buffer (Sigma) containing protease/ phosphatase inhibitor cocktail (Sigma). Quantity of $10 \mu \mathrm{g}$ of each lysate was separated on 10\% SDS-polyacrylamide gel and transferred to PVDF membrane. Immunoblot analysis was performed by using anti-p-tau (Ser199), antip-tau (Ser396), and anti- $\beta$-tubulin antibodies. All antibodies were purchased from Abcam. Band intensity was quantified using Image J software (NIH).

\section{Generation of glioblastoma-xenograft model}

All animal experiments were approved by the Korea Institute of Science and Technology, and the experimental protocols were carried out in accordance with the approved guidelines by the Institutional Animal Care and Use Committee of the Korea Institute of Science and Technology.

To generate the glioblastoma-xenograft mouse model, U87 cells were suspended in PBS and $5 \times 10^{5}$ of U87 cells were injected into the striatum of each $\mathrm{BALB} / c$ nude mouse $(n=7)$. As a control, PBS was injected $(n=5)$. After 4 weeks of tumorigenesis, the mice were perfused and the brains were fixed with $4 \%$ paraformaldehyde. Frozen brain tissues were prepared at $30-\mu \mathrm{m}$ thicknesses with a cryostat (Leica). 


\section{Stereotaxic injection of sCD44 into MAPT tg mice}

Four-month-old tau transgenic (MAPT*P301L) mice expressing human tau containing P301L mutation were used for sCD44 injection ${ }^{22}$. Based on the atlas of Frankin and Paxions ${ }^{23}, 9 \mu \mathrm{g}$ of purified sCD44 (dissolved in $8 \mu \mathrm{L}$ of PBS) was injected into the hippocampus of each mouse brain at AP: $-2.06 \mathrm{~mm}$ and ML: $-2.0 \mathrm{~mm}$ by pressure injection at $0.2 \mu \mathrm{L} / \mathrm{min}(n=6)$. Quantity of $8 \mu \mathrm{L}$ of PBS was injected as a control $(n=5)$. Two weeks following the injection, the mice were perfused and the brains were fixed with $4 \%$ paraformaldehyde. Frozen brain tissues were prepared at $30-\mu \mathrm{m}$ thickness with a cryostat (Leica).

\section{Immunofluorescence analysis}

For immunofluorescence analysis, brain slices from glioblastoma-xenograft mice and sCD44-injected MAPT tg mice were stained with anti-AT8 (pS202/T205, 1:200, Abcam), anti-AT100 (pT212/S214, 1:200, Abcam), anti-pTau (Ser199, 1:200, Abcam), and anti-NeuN (1:500, Millipore) antibodies. Nuclei were counterstained with Hoechst. To analyze the distribution of CD44, the brain slices were stained with Cy5-conjugated anti-sCD44 antibody (1:500, Abcam) and anti-iCD44 (1:500, Cell Signaling Technology). Fluorescent images were taken by using Operetta (PerkinElmer). To calculate the percentage of phospho-tau positive neurons, the number of total neurons and phospho-tau positive neurons were counted in the cortical, striatum, and hippocampal regions $\left(\mathrm{mm}^{2}\right)$. Error bars indicate the standard deviation of three different hippocampal regions. The statistical analysis was performed via paired $t$-test and the significance was considered at a level of $p<0.05$.

Human tissue samples and immunohistochemistry

This study was approved by the institutional review board (IRB) of the Seoul National University College of Medicine and Hospital. The IRB, due to the retrospective nature of this study, waived the informed consent requirement from the participants. Human brain tissues were surgically extracted from glioblastoma patients $(n=$ 6) at the Seoul National University Hospital. FFPE (10\% neutral buffered formalin, routinely processed, and paraffin embedded) tissue Section ( $3 \mu \mathrm{m}$ thick) were cut for immunohistochemistry. The immunohistochemical stain was carried out by using a Ventana autostainer. After deparaffinization with EZ prep, quenching of endogenous peroxidase activity and antigen unmasking were performed by incubation with cell conditioning 1 at about $100{ }^{\circ} \mathrm{C}$ for $32 \mathrm{~min}$. The sections were stained with antiAT8 (1:200, Abcam) antibody. The reaction products were then developed by immersing the sections in 3.3'diaminobenzidine tetrahydrochloride solution containing $0.03 \% \mathrm{H}_{2} \mathrm{O}_{2}$.

\section{Fast protein liquid chromatography (FPLC)}

Quantity of $40 \mathrm{~mL}$ of HS683-, U87-, and T98Gconditioned media were collected and the $30 \sim 50 \mathrm{kDa}$ fractions of the conditioned media were sequentially fractioned by using Centricon filters, of which molecular weight cutoffs are 30 and $50 \mathrm{kDa}$. Then, $1 \mathrm{~mL}$ of each 30-50 kDa fraction was loaded onto a Superdex-75 10/ 300 GL High performance column (GE Healthcare) and separated by FPLC using a ÄKTAFPLC system (GE Healthcare). The separated proteins were eluted with PBS buffer $(0.5 \mathrm{ml} / \mathrm{min})$.

\section{siRNA transfection and analysis}

SPARC, CD44 and LDHA siRNAs were purchased from OriGene Technologies, Inc. For siRNA transfection, U87 cells $\left(5 \times 10^{4}\right.$ cells/well) were plated on 6 -well plates in Opti-MEM (Invitrogen) and transfected with each of the siRNAs by using Lipofectamine 2000 (Invitrogen). Universal scrambled siRNA duplex was purchased from OriGene Technologies ( $\mathrm{r}\left(5^{\prime}\right.$-CGUUAAUCGCGUAUAAUACGCGU-3')AT). Scrambled siRNA was used as a control. Eighteen hours after transfection, the medium was replaced with fresh growth medium (DMEM containing $10 \%$ FBS with antibiotics). Seventy-two hours after transfection, each medium was collected for tau$\mathrm{BiFC}$ analysis and siRNA transfected U87 cells were collected for immunoblot analysis with anti-CD44 antibody (Abcam).

\section{Results}

Tangle-bearing neurons are observed in the glioblastoma brain

To investigate glioblastoma-activated tau pathology, glioblastoma-xenograft mice were generated by injecting U87 cells into the brain of BALB/c nude mice $(n=7)$. PBS-injected mice were used as the control $(n=5)$. After 1 month of gliomagenesis, U87-xenograft mice were killed and the brain tissue sections were examined. Bright-field images indicate tumors formed in the striatum (Fig. 1a). Cresyl violet stain showed massive degeneration of cortical neurons adjacent to the tumor, marked with a red line in Fig. 1a (Fig. 1b). The number of cortical neurons was reduced to $53 \%$, compared to that observed in the PBS-injected group (Fig. 1c). Immuno-fluorescence analysis with AT8 and AT100 was followed to investigate the association of tau pathology with glioblastoma. AT8 and AT100 antibodies are known for their selectivity for paired helical filaments (PHFs), the late stage of tau aggregation (Fig. 1d, e). Neuronal cells were counterstained with NeuN antibody. AT8 and AT100-positive neurons were observed in the tumor region and in the cortical region adjacent to the tumor. The corresponding brain regions of PBS-injected mice did not show noticeable responses to AT8 or AT100. The number of AT8- 
A

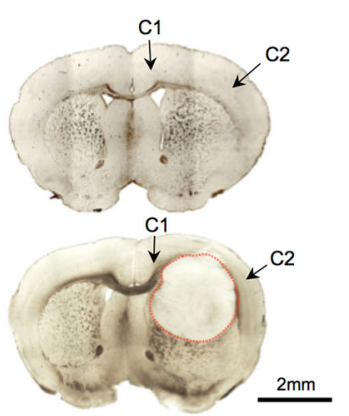

D
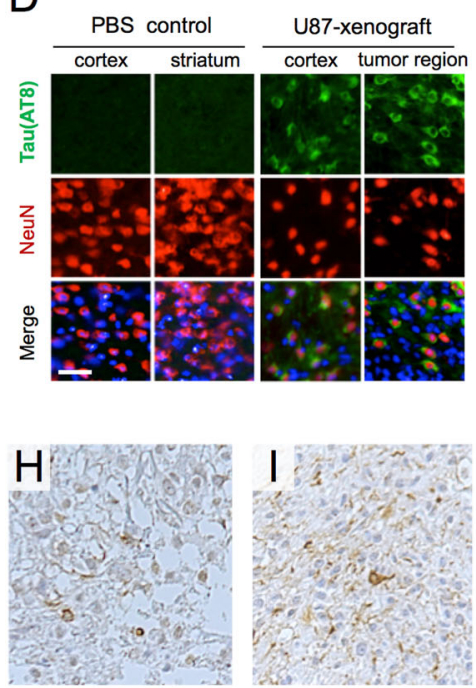

B

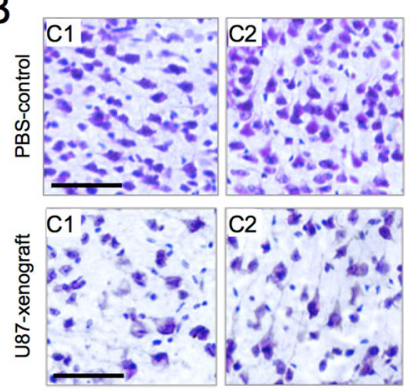

$\mathrm{E}$
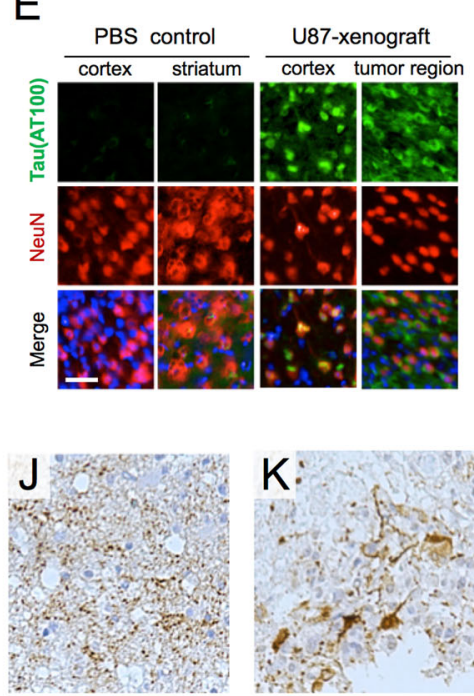

C

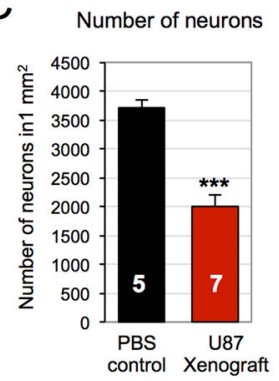

$\mathrm{F}$

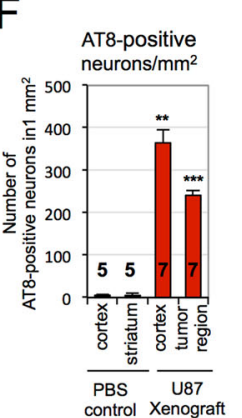

G AT100-positive
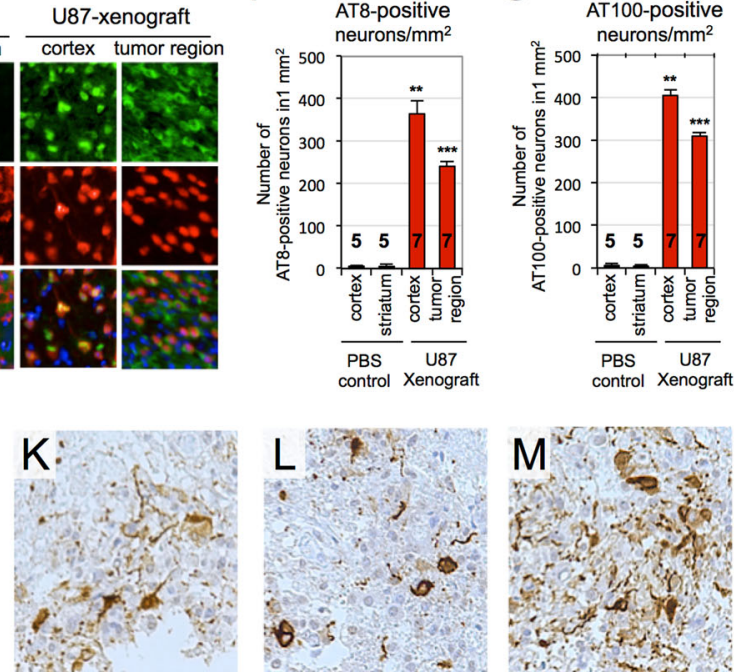
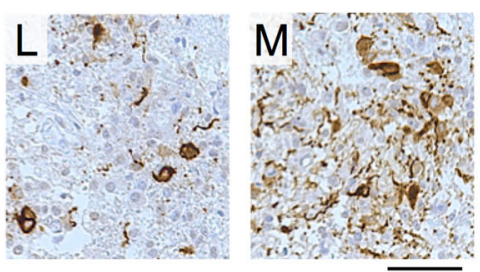

Fig. 1 Elevated tau pathology in U87-xenograft model and primary glioblastoma tissues. a Bright field images of PBS-injected (up) and U87xenograft (down) brain tissues. U87 cells were stereotaxically injected into the striatum of BALB/C nude mice brain $(n=7)$. PBS-injected mice were used as a negative control $(n=5)$. After 4 weeks of tumorigenesis, brains were extracted and tissue sections were prepared. b Cresyl violet stained tissues of PBS-control and U87-xenograft. Degenerating neurons were observed in the cortical regions, designated as C1 and C2 in the U87-xenograft brain. Scale bar, $250 \mu \mathrm{m}$. c Number of neurons in the cortex. Bars indicate both the mean and standard deviation of the mean, ${ }^{* * *} P<0.001$. d, e Tau immunofluorescent images with anti-AT8 (d) and anti-AT100 (e) antibodies. Neuronal nuclei were counterstained with NeuN. Scale bar, $200 \mu \mathrm{mm}$. f, $\mathbf{g}$ Numbers of AT8- and AT100-positive neurons in $1 \mathrm{~mm}^{2} . \mathbf{h}-\mathbf{m}$ AT8 immuohistochemical stained images of human glioblastoma tissues $(n=6)$. Paraffin-embedded tissue sections were stained with anti-AT8 antibody (brown). Nuclei were counterstained with Hematoxylin (blue). Scale bar, $400 \mu \mathrm{m}$

positive neurons was increased by 25 -fold in cortex and 16 -fold in tumor region, compared to that of PBS-injected brains (Fig. 1f). The number of AT100-positive neurons was also increased by 23 -fold in cortex and 17 -fold in tumor region (Fig. 1g). Additional time-course experiment indicated that tau pathology was primarily detected in tumor region after 2 weeks, and secondarily detected in cortex after 4 weeks of tumorigenesis. (Figure S1). The glioblastoma-xenograft tissues clearly showed the elevated tau pathology.

To validate the elevated tau pathology in primary glioblastoma tissues, we examined tau pathology in human glioblastoma tissues surgically removed from the patients with glioblastoma $(n=6)$ who have not been diagnosed neurodegenerative disorders. AT8 immuno-stains showed highly variable astrocytic ${ }^{24}$ and neuronal tau pathology in the glioblastoma tissues (Fig. 1h-m). Astrocytic tau fibrils were observed in all the glioblastoma tissues extracted from diverse brain regions (Fig. $1 \mathrm{~h}-\mathrm{j}$ ). Neuronal tau inclusions were observed in three cases, of which tumors were extracted from frontal or temporal lobes (Fig. $1 \mathrm{k}-\mathrm{m}$ ). Although the degree of tau pathology was diverse depending on the tumor region, our result clearly shows the elevated tau pathology in human glioblastoma tissues.

\section{Glioblastoma-secretome activates tau hyper- phosphorylation and aggregation}

We exposed tau-aggregation sensor cells, named taubimolecular fluorescence complementation (tau-BiFC) to 

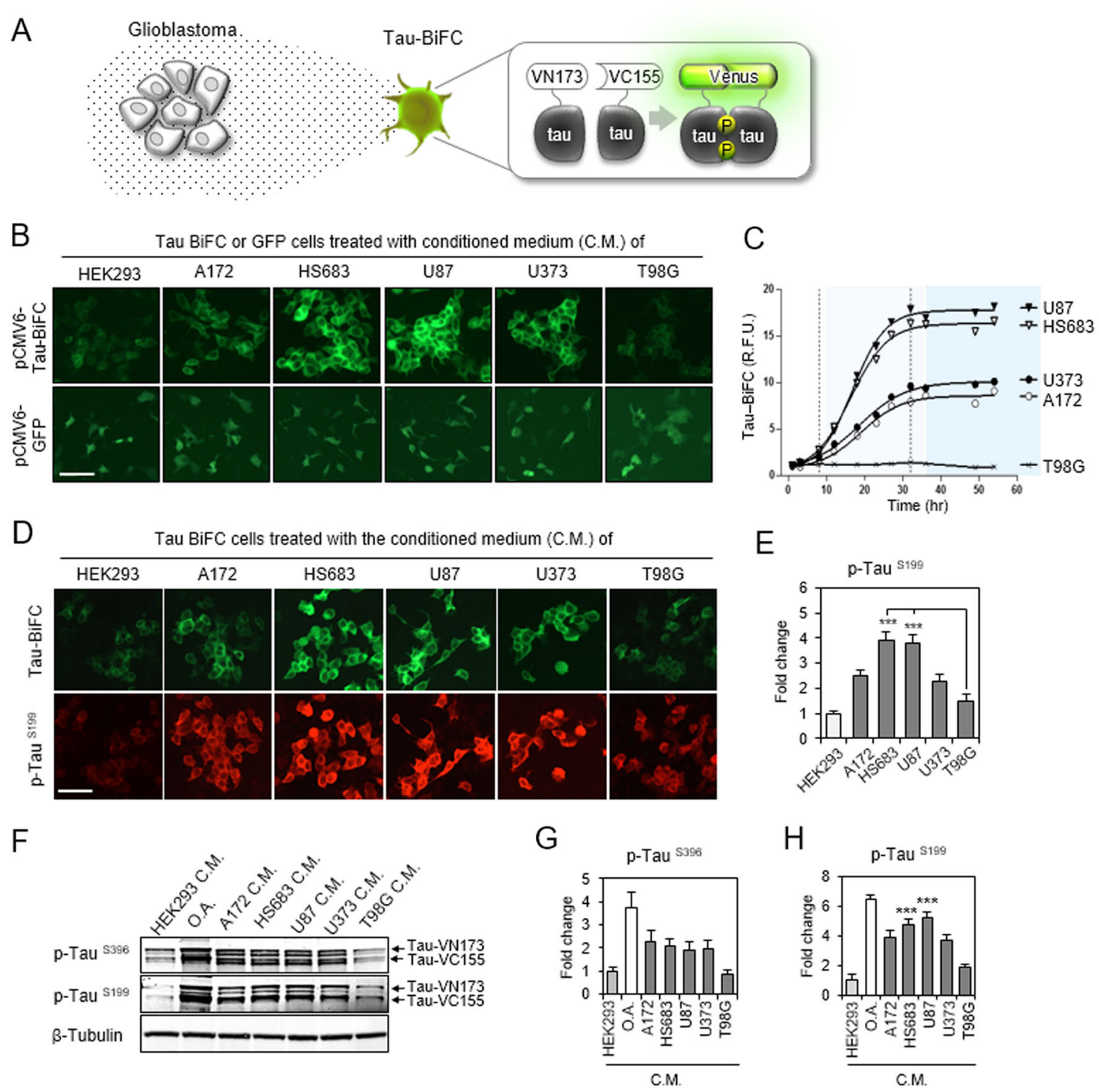

Fig. 2 Glioblastoma activates tau hyper-phosphorylation and aggregation. a Illustration of glioblastoma-secretome effect on tau-BiFC sensor cells. As a fluorescence turn-on sensor, Venus fluorescence turns on only when tau assembles together. $\mathbf{b}$ The effect of glioblastoma conditioned media (C.M.) on tau aggregation. Tau-BiFC cells were treated with glioblastoma (A172, HS683, U87, U373, or T98G) conditioned media or HEK293conditioned medium for $48 \mathrm{~h}$. Stable cells expressing GFP were tested as a negative control, indicating that glioblastoma conditioned media did not activate CMV-promoter mediated gene expression. Scale bar, $100 \mu \mathrm{m}$. c Quantification of tau-BiFC fluorescence intensity. Error bars represent the standard deviations of three independent experiments. d, e Tau immuno-fluorescence images with p-Tau (Ser199) antibody and the quantification. The significance of the experiments was determined with Student's $t$-test. ${ }^{* *} p<0.001$. $\mathbf{f}$ Immuno-blot analysis of tau phosphorylation with $p$-Tau (Ser396) or anti-p-Tau (Ser199). For the immuno-blot analysis, tau-BiFC cells were treated with glioblastoma-C.M for 24 h. HEK293-conditioned medium was used as a negative control and okadaic acid (O.A.) was used as a positive control. g, h Quantification of p-Tau (Ser396) and p-Tau (Ser199). Error bars represent the standard deviations of three independent experiments. The significance of the experiments was determined with Student's t-test. ${ }^{* * *} p<0.001$

conditioned media collected from five different glioblastoma cells (A172, HS683, U87, U373, and T98G) (Fig. 2a) ${ }^{19,25,26}$. Tau-BiFC cells are HEK293-derived stable cell line, which constitutively expresses tau-BiFC vectors. In tau-BiFC system, tau is fused to each of the nonfluorescent $\mathrm{N}$ - and C-terminal compartments of a Venus protein. As a fluorescence turn-on sensor, Venus fluorescence turns on, only when tau assembles together. When tau-BiFC cells were treated with glioblastoma conditioned media, tau-BiFC responses were significantly increased by all glioblastoma conditioned media except T98G (Fig. 2b). HEK293-conditioned medium was used as 

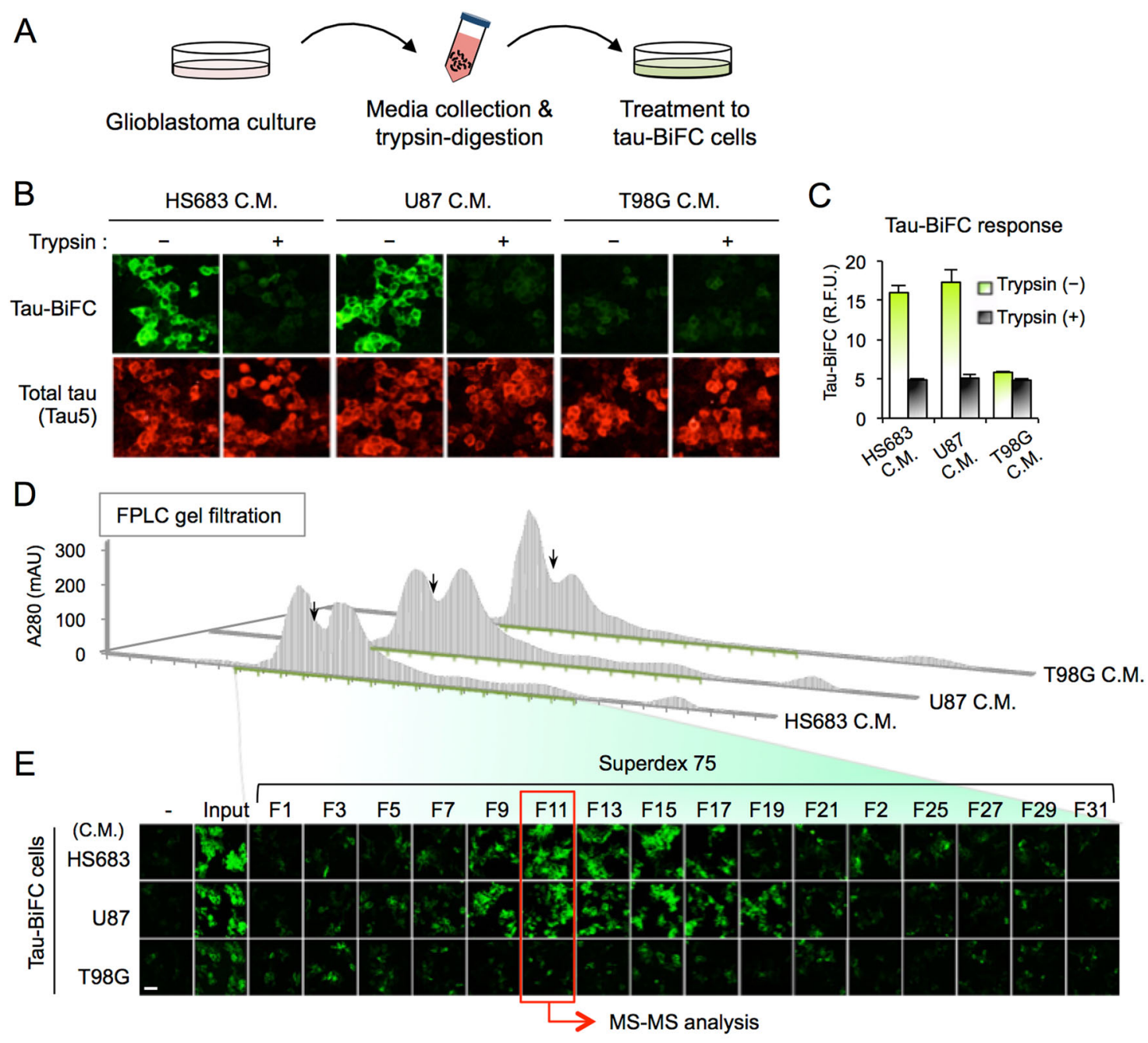

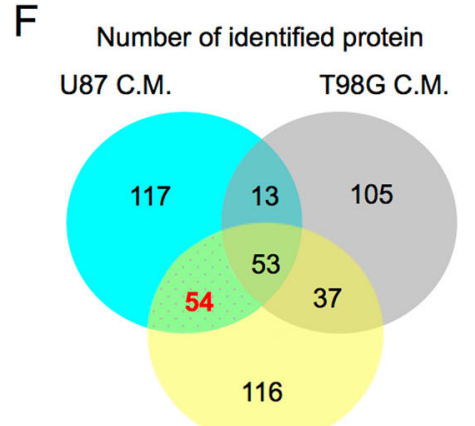

HS683 C.M.

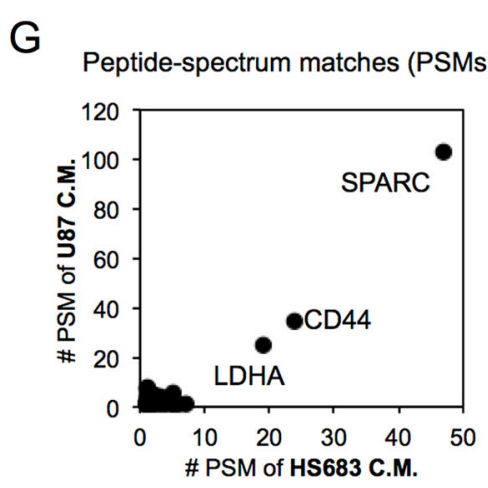

$\mathrm{H}$

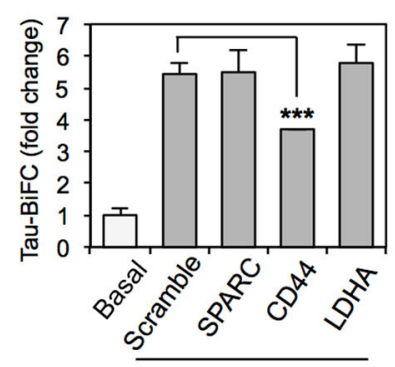

U87 siRNA knock-down

Fig. 3 Secretome analysis identifies CD44. a Experimental scheme of collecting glioblastoma C.M., trypsin-digestion, and treatment to tau-BiFC cells. b Tau-BiFC responses and tau immunofluorescence images with anti-Tau5 antibody upon the treatment of trypsin-digested or non-digested glioblastoma C.M. Scale bar, $100 \mu \mathrm{m}$. c Quantification of tau-BiFC intensities by using a Harmony 3.1 software. Error bars represent the standard deviation of three independent experiments. d Separation of glioblastoma (HS683, U87, or T98G) C.M. by using FPLC with a Superdex75 gel filtration column. e Tau-BiFC responses upon the treatment of odd-numbered FPLC fractions. Fraction 11 of HS683 and U87 showed the highest tau-BiFC responses and was subjected to mass-spectrometry analysis. Scale bar, $100 \mu \mathrm{m}$. $\mathbf{f}$ Diagram showing the number of identified proteins. A total of 495 proteins was identified from the fraction 11 of HS683-, U87-, and T98G-C.M., and 54 proteins were identified both in HS683- and U87-C.M. but not in T98G. C.M. g Peptide-spectrum matches (PSMs) of 54 proteins. PSM indicates that LDHA, CD44, and SPARC are the three major proteins. $\mathbf{h}$ Target validation by siRNA knockdown. U87 cells were transfected with LDHA, CD44, or SPARC siRNA. After $72 \mathrm{~h}$ of transfection, U87-conditioned media were collected and treated to tau-BiFC cells. Error bars represent the standard deviation from four independent experiments. The significance of the experiments was determined with Student's $t$-test. ${ }^{* *} p<0.001$ 
a negative control. Intriguingly, the level of tau-BiFC responses varied depending on the glioblastoma celltypes. HS683- and U87-conditioned media increased tauBiFC fluorescence dramatically by showing 13.3- and 14.6-fold increases (Fig. 2c). A172- and U373-conditioned media-induced moderate tau-BiFC responses by showing 6.5- and 7.8-fold increases. To exclude any possibility of non-specific CMV promoter activation induced by glioblastoma-conditioned media, HEK293 cells expressing green fluorescence protein (GFP) were tested as a control (Fig. 2b). The results indicate that glioblastomaconditioned media did not affect GFP expression.

Immunofluorescence stain with p-Tau (Ser199) antibody showed that cellular distribution of tau-BiFC fluorescence matches tau phosphorylation patterns (Fig. 2d). Tau phosphorylation levels were increased 3.9- and 3.8fold by HS683- and U87-conditioned media, 2.5- and 2.3fold by A172- and U373-conditioned media (Fig. 2e), while total tau expression levels were similar (Figure S2). Again, T98G-conditioned media did not induce noticeable changes in tau phosphorylation. Immunoblot analysis confirmed the increased level of tau phosphorylation in tau-BiFC cells that were treated with A172-, HS683-, U87-, and U373-conditioned media (Fig. 2f). The pattern of tau phosphorylation at Ser199 closely matches tauBiFC responses (Fig. 2g, h). Okadaic acid (O.A.) was used as a positive control in inducing tau hyperphosphorylation. The in vitro results demonstrate that glioblastoma-secretome could activate abnormal tau hyperphosphorylation and aggregation.

\section{Secretome-analysis identifies SCD44 as a responsible protein}

Next, to identify glioblastoma-secretome responsible for inducing tau pathology, we conducted a series of secretome-analyses. First, glioblastoma-conditioned media were digested with trypsin to determine whether the responsible secretome is a protein(s) (Fig. 3a). When tau-BiFC cells were treated with trypsin-digested glioblastoma conditioned media, trypsin-digested HS683- and U87-conditioned media were no longer effective, indicating that a protein(s) is the responsible factor (Fig. 3b, c). Total tau expression levels were similar in both cases. To verify the approximate size of the responsible protein (s), HS683- and U87-conditioned media were sequentially fractionated by Centricon with various molecular weight cutoffs $(10,30,50$, and $100 \mathrm{kDa}$ ) (Figure S3). T98G conditioned medium was used as a negative control. Only $30-50 \mathrm{kDa}$ fractions-induced a tau-BiFC response indicating that the size of the responsible protein is between 30 and $50 \mathrm{kDa}$. Then, the enriched $30-50 \mathrm{kDa}$ fraction was further separated by using FPLC) with Superdex75 gel filtration column (Fig. 3d). Then, tau-BiFC cells were treated with every odd-numbered fraction (Fig. 3e). The
tau-BiFC response indicated that fraction 11 of both HS683- and U87-conditioned media is the most effective fraction enriched with the responsible protein(s) (Figure S4).

For protein identification, fraction 11 of HS683-, U87-, or T98G-conditioned medium was subjected to mass spectrometry analysis (Raw data are available via ProteomeXchange with identifier PXD007969; Supplementary Note 1$)^{27}$. As a result, a total of 495 proteins was identified; 260 from HS683, 237 from U87, and 208 from T98Gconditioned media (Fig. 3f). Among those, 54 proteins overlapped both in HS683 and U87 fractions but not in the T98G fraction used as a negative control (Table. S1). The 54 proteins were further checked for their peptidespectrum matches (PSMs) (Fig. 3g). Based on PSMs values, secreted protein acidic and rich in cysteine (SPARC), CD44, and L-lactate dehydrogenase A (LDHA) were identified as the most "abundant" proteins in fraction 11 . To validate the function of the proteins, a siRNA knockdown experiment was conducted. U87 cells were transfected with siRNAs against SPARC, CD44, or LDHA. Eighteen hours after transfection, transfection media were replaced with normal growth medium. After $48 \mathrm{~h}$, each conditioned media was collected. Among the tested, only CD44-siRNA-conditioned medium showed decreased tau-BiFC response by presenting 32\% reduced BiFC fluorescence (Fig. 3h and Figure S5). Neither SPARC- nor LDHA-siRNA conditioned media decreased the tau-BiFC response compared to the scrambled siRNA conditioned medium. The siRNA experiment suggests that CD44 might be one of the responsible proteins inducing tau aggregation. CD44 $(\sim 85 \mathrm{kDa})$ has been designated as a cell surface marker for the malignant tumor participating in tumor cell adhesion and progression ${ }^{28,29}$. During tumor cell migration, the extracellular domain of CD44 (sCD44; $32 \mathrm{kDa}$ ) is known to be released from the glioblastoma cell surface $^{30,31}$. Although the role of sCD44 is still unclear, the secretion of sCD44 has been reported in $58 \%$ of glioblastoma patients ${ }^{31}$.

\section{SCD44 activates tau pathology in vitro and in vivo}

Next, we evaluated the expression level of CD44 in U87, HS683, and T98G cells. Immunofluorescence stain with an intracellular CD44 antibody (iCD44) showed highly expressed CD44 on the plasma membrane of HS683 and U87 cells (Fig. 4a). In comparison, CD44 was not detected in T98G cells. Immunoblot analysis also displayed CD44 expression levels of each glioblastoma cell lines (Fig. 4b). Then, we quantified soluble CD44 (sCD44) levels in the glioblastoma-conditioned media (Fig. 4c). As expected, high concentrations of sCD44 were detected in HS683and U87-conditioned media $(83.5 \mathrm{ng} / \mathrm{mL}$ and $61.1 \mathrm{ng} / \mathrm{mL}$, respectively). A low concentration of $\mathrm{sCD} 44$ was detected in T98G-conditioned medium $(7.2 \mathrm{ng} / \mathrm{mL})$. To ensure the 
A

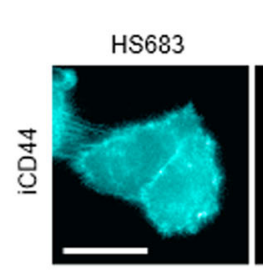

D

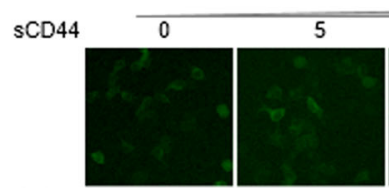

Chi3L1

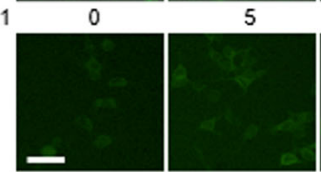

U87
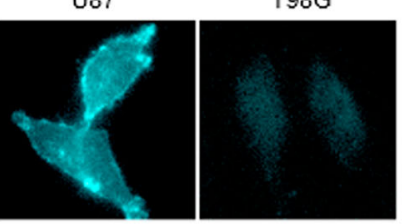

B

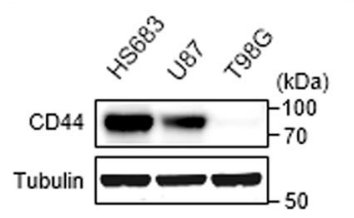

C

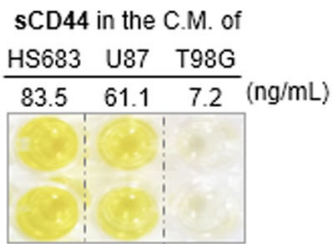

E

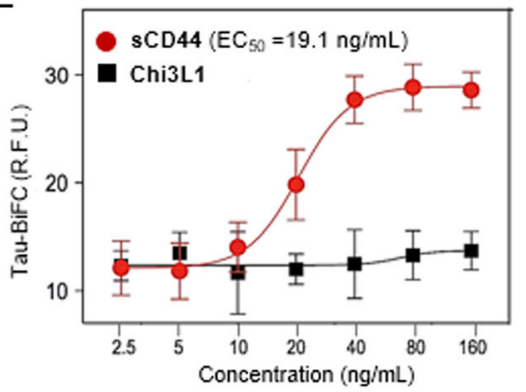

F

PBS

SCD 44
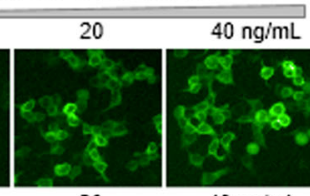

10 20 $40 \mathrm{ng} / \mathrm{mL}$
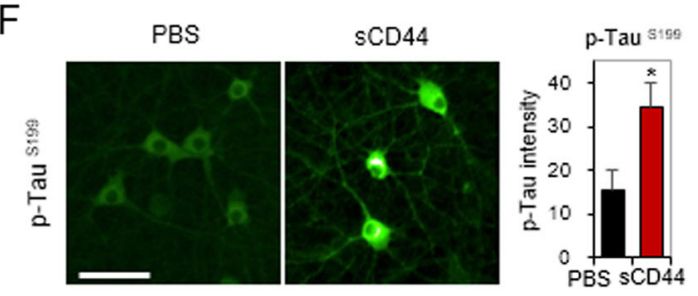

G
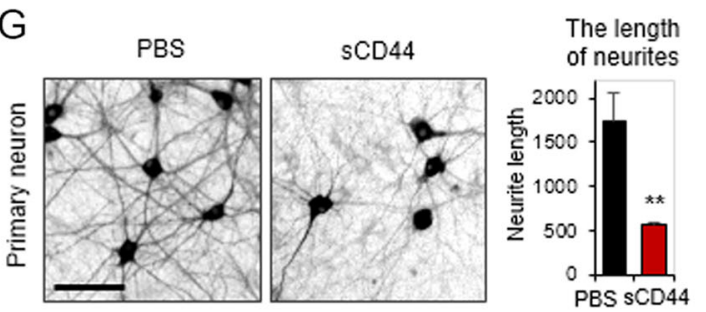

$\mathrm{H}$
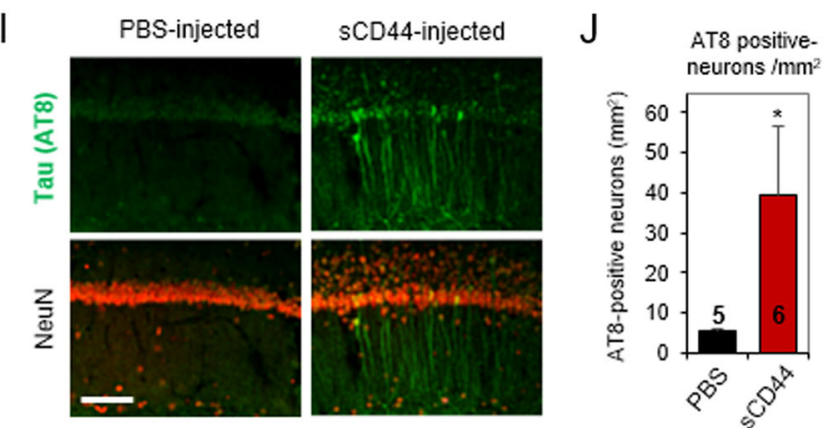

Fig. 4 sCD44 induces tau phosphorylation and aggregation. a CD44-immunofluorescence images of HS683, U87, and T98G cells. Cells were fixed and stained with anti-intracellular CD44 (iCD44) antibody. Scale bar, 50 m. b CD44 immuno-blot analysis. HS683, U87, and T98G cell lysates were prepared and analyzed with anti-iCD44 or anti-tubulin antibodies. c SCD44 levels in HS683-, U87-, and T98G-C.M. were quantified by using a human SCD44 ELISA assay. Experiments were performed in duplicate. $\mathbf{d}$ Tau-BiFC responses upon the treatment of SCD44 or Chi3L1 at various concentration. Scale bar, $100 \mu \mathrm{m}$. e Quantification of tau-BiFC-fluorescence intensity. A Prism's non-linear regression analysis was used to measure the $\mathrm{EC}_{50}$ value. The $\mathrm{EC}_{50}$ of sCD44 is $19.1 \mathrm{ng} / \mathrm{mL}$. f Tau-immunofluorescence images of primary hippocampal neurons upon the treatment of sCD44. Rat embryonic hippocampal neurons were treated with sCD44 protein $(120 \mathrm{ng} / \mathrm{mL})$ at 7 days in vitro and incubated for $48 \mathrm{~h}$. Scale bar, $100 \mu \mathrm{m}$. p-Tau (Ser 199) intensity was measured by using a Harmony 3.1 software. ${ }^{*} p<0.05$. $\mathbf{g}$ Live hippocampal neurons stained with $\mathrm{NeuO}^{21}$. After $48 \mathrm{~h}$ of $\mathrm{sCD} 44$

$(120 \mathrm{ng} / \mathrm{mL})$ treatment, hippocampal neurons were stained with NeuO and imaged. The maximal length of neurites was quantified by the Harmony 3.1 software. Scale bar, $100 \mu \mathrm{m}$. Error bars represent the standard deviation of three independent experiments. ${ }^{* *} p<0.01 . \mathbf{h}$ sCD 44 was stereotaxically injected to the hippocampus of 4-month-old tau transgenic (MAPT*P301L) mice $(n=6)$. i Experimental scheme of sCD44 injection to the hippocampus of 4-month-old tau transgenic (MAPT*P301L) mice $(n=6)$. Scale bar, $50 \mu \mathrm{m}$. j A number of AT8-positive neurons was calculated in the hippocampal region (cells $/ \mathrm{mm}^{2}$ ) of the SCD44- $(n=6)$ or PBS-injected $(n=5)$ mice brain tissues. The significance of the experiments was determined by Student's t-test. ${ }^{*} p<0.05$ 


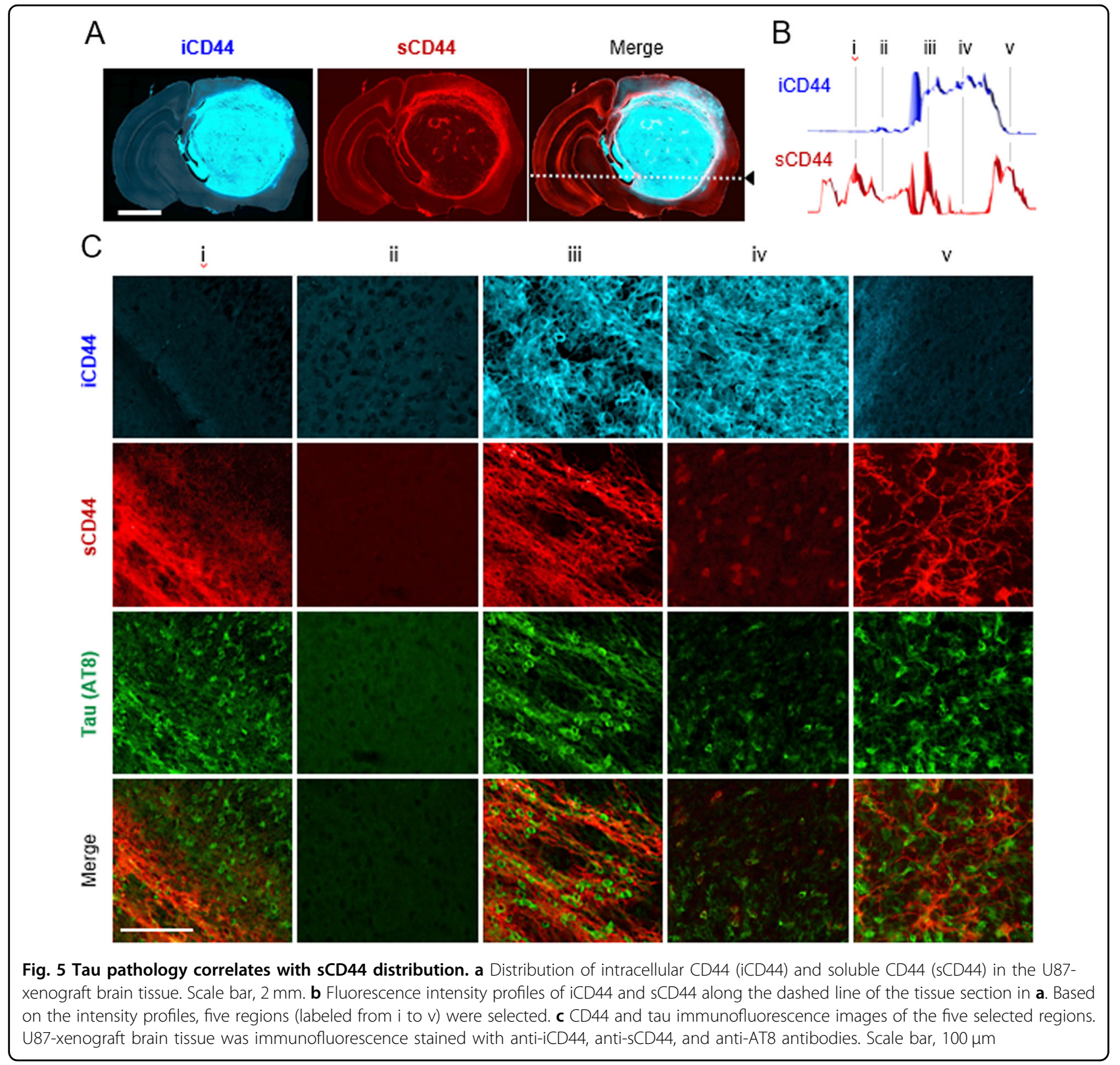

direct effect of sCD44 in inducing tau pathology, tau-BiFC cells were treated with purified human sCD44 (Fig. 4d). Chi3L1, a glioblastoma-secretome marker ${ }^{32}$, was treated as a control. As expected, the tau-BiFC response was significantly increased by the treatment of SCD44 $\left(\mathrm{EC}_{50}=\right.$ $19.1 \mathrm{ng} / \mathrm{mL}$ ) but not by the treatment of Chi3L1 (Fig. 4e). In both cases, total tau expression levels were unchanged (Figure S6). Upon the treatment of sCD44, tau hyperphosphorylation pattern was also correlated with the increased tau-BiFC signals. The result showed that SCD44 could activate tau phosphorylation and tau aggregation.

To investigate the effect of sCD44 on neuronal integrity, sCD44 was treated to primary neurons. Primary hippocampal neurons were isolated from day-18 rat embryos.
After 7 days in vitro culture, sCD44 (120 ng/mL) was treated to the hippocampal neurons. Anti-p-Tau(Ser199) antibody stain showed that tau phosphorylation was significantly induced in the soma of hippocampal neurons treated with $\mathrm{sCD} 44$, by showing a 2.3 -fold increase (Fig. 4f). Next, to evaluate neuronal integrity, primary neurons were stained with $\mathrm{NeuO}$, a neuron selective fluorescence probe, and analyzed ${ }^{21}$. After $48 \mathrm{~h}$ of sCD44 treatment, primary hippocampal neurons were significantly degenerated by showing $68 \%$ shortened neurites (Fig. 4g). These results clearly indicate that sCD44 activates tau pathology, leading to neuronal degeneration.

Next, to validate the effect of sCD44 in vivo, purified sCD44 was directly injected into the hippocampus of 4- 
month-old tau transgenic (MAPT*P301L) mouse brains $(n=6 \text {, Fig. } 4 \mathrm{~h})^{22}$. Two weeks after sCD44 injection, the brains were extracted and the brain tissue sections were prepared for immune-fluorescence stain. Anti-AT8 antibody staining showed abnormal tau hyperphosphorylation in the hippocampus especially near the sCD44 injection site (Fig. 4i). Quantitative analysis found a statistically significant increase (by $36 \%$ ) in the number of AT8-positive neurons in the hippocampal region $\left(\mathrm{mm}^{2}\right)$ of sCD44-injected mice brains $(n=6)$, as compared to PBS-injected mouse brains $(n=5)$ (Fig. 4j). The results clearly demonstrate that $\mathrm{sCD} 44$ contributes to activating tau pathology and neuronal degeneration.

\section{Tau pathology correlates with sCD44 distribution in the glioblastoma brain}

Next, we scrutinized CD44 distribution in the brain of U87-xenograft mice. Immuno-fluorescence stain with anti-intracellular CD44 (iCD44) antibody showed highdensity tumor mass in the U87-injected region. In contrast, sCD44 immuno-stain showed highly enriched sCD44 fragments in the edge of the tumor (Fig. 5a, b). This result supports that CD44 cleavage and secretion occur massively on the leading edge of the tumor. Interestingly, sCD44 was also detected in the opposite hemisphere of the brain, which is far from the tumor region. If sCD44 could propagate or circulate in the brain, tau pathology could be activated in any region of the brain. To investigate the association between $\mathrm{sCD} 44$ and tau phosphorylation, five horizontal regions of the brain were selected (Fig. 5c). When stained with an AT8 antibody, AT8-positive neurons were observed in not only the glioblastoma regions but also in the opposite hemisphere of the brain, where sCD44 is highly enriched. AT8positive tau pathology was strongly correlated with the distribution of sCD44.

\section{Discussion}

As a well-known marker for the malignant tumors, CD44 plays critical roles in tumor cell adhesion and migration; the extracellular domain of CD44 binds to hyaluronic acid, a component of the extracellular matrix, anchoring the cancer cell to the extracellular matrix ${ }^{30}$. For tumor cell migration, the extracellular domain of CD44 is cleaved by membrane-associated proteases. The elevated level of sCD44 has been detected in 58\% of gliomas, $67 \%$ of breast carcinomas, $45 \%$ of non-small cell lung carcinomas, and $90 \%$ of colon carcinomas, indicating the malignancy of the tumor ${ }^{31}$. So far, it is unclear whether sCD44 has a specific role in the brain. Here, our results demonstrate that $\mathrm{sCD} 44$ activates abnormal tau hyper-phosphorylation and aggregation, which suggests a role of this polypeptide in brain neurodegeneration.
Clinical studies showed that $50 \%$ of the long-term survivors of glioblastoma exhibit cognitive declines and 30\% of the long-term survivors become demented ${ }^{33}$. So far, it is generally considered that the aggressive tumor treatment including surgical resection combined with chemoand radiation therapy are the major causes of neurodegeneration in glioblastoma patients. In addition, glioblastoma generates diverse metabolic and mechanical stresses in the brain ${ }^{34}$. Especially, excitotoxic concentrations of glutamate is secreted from glioblastoma cells, leading to neuronal cell death ${ }^{35}$. Also, physical constraints of glioblastoma generate shear stresses ${ }^{36,37}$, which have a potential to trigger tau pathology in the brain. Here, our findings suggest that glioblastoma-secretome could initiate tau pathology in the brain and demonstrate that sCD44 plays a critical role as a pathological link between glioblastoma and neurodegeneration.

\section{Acknowledgements}

We express our deep gratitude to Prashant Kaushal for English-proof. This work was supported by grants from the following funding agencies: the National Research Council of Science and Technology (NST) granted by the Ministry of Science, ICT and Future Planning (MSIP) (No. CRC-15-04-KIST); the Brain Research Program through the National Research Foundation of Korea (NRF) funded by the MSIP (2016M3C7A1913844); the BioMedical Research Program thorugh the NRF funded by the MSIP (2017M3A9F9030559); the Basic Science Research program through the NRF funded by the Ministry of Education (2017R1A6A3A04012384); and the Research Hospital Program through the Korea Health Industry Development Institute (KHIDI) funded by the Korean Ministry of Health and Welfare (HI14C3484).

\section{Author details \\ 'Korea Institute of Science and Technology (KIST), Brain Science Institute, Convergence Research Center for Diagnosis, Treatment and Care System of Dementia, Seoul 02791, Republic of Korea. ${ }^{2}$ Department of Biotechnology, Translational Research Center for Protein Function Control, College of Life Science and Biotechnology, Yonsei University, Seoul 03722, Republic of Korea. ${ }^{3}$ Korea Institute of Science and Technology (KIST), Biomedical Research Institute, Center for Theragnosis, Seoul 02791, Republic of Korea. ${ }^{4}$ Korea Institute of Science and Technology (KIST), Brain Science Institute, Center for Bio-Microsystems, Seoul 02791, Republic of Korea. ${ }^{5}$ Department of Pathology, Seoul National University Hospital, Seoul 110-744, South Korea. Institut Pasteur Korea, Technology Development Platform, Sungnam-si, Gyeonggi-do 13488, Republic of Korea. ${ }^{7}$ Biological Chemistry, University of Science and Technology (UST), Daejeon, South Korea}

\section{Conflict of interest}

The authors declare that they have no conflict of interest.

\section{Publisher's note}

Springer Nature remains neutral with regard to jurisdictional claims inpublished maps and institutional affiliations.

Supplementary information accompanies this paper at https://doi.org/ 10.1038/s12276-017-0008-7.

Received: 3 July 2017 Revised: 19 October 2017 Accepted: 24 October 2017.

Published online: 6 April 2018

\footnotetext{
References

1. Schwartzbaum, J. A., Fisher, J. L., Aldape, K. D. \& Wrensch, M. Epidemiology and molecular pathology of glioma. Nat. Clin. Pract. Neurol. 2, 494-503 (2006).
} 
2. Ohgaki, H. \& Kleihues, P. Epidemiology and etiology of gliomas. Acta Neuropathol. 109, 93-108 (2005).

3. DeAngelis, L. M. Brain tumors. N. Engl. J. Med. 344, 114-123 (2001).

4. Claes, A., Idema, A. J. \& Wesseling, P. Diffuse glioma growth: a guerilla war. Acta Neuropathol. 114, 443-458 (2007).

5. Schiffer, D. Brain tumors: biology, pathology, and clinical references. (Springer Science \& Business Media, NY, USA, 1997).

6. Miotto, E. C. et al. Cognitive impairments in patients with low grade gliomas and high grade gliomas. Arch. Neuropsychiatry 69, 596-601 (2011).

7. Hahn, C. et al. Prospective study of neuropsychologic testing and quality-oflife assessment of adults with primary malignant brain tumors. Int. J. Radiat Oncol. Biol. Phys. 55, 992-999 (2003).

8. Liu, R., Page, M., Solheim, K., Fox, S. \& Chang, S. M. Quality of life in adults with brain tumors: current knowledge and future directions. Neuro. Oncol. 11, 330-339 (2009).

9. Paulus, W. \& Tonn, J. C. Interactions of glioma cells and extracellular matrix. J. Neuro Oncol. 24, 87-91 (1995).

10. Demuth, T. \& Berens, M. E. Molecular mechanisms of glioma cell migration and invasion. J. Neuro Oncol. 70, 217-228 (2004)

11. Polisetty, R. V. et al. Glioblastoma cell secretome: analysis of three glioblastoma cell lines reveal 148 non-redundant proteins. J. Proteom. 74, 1918-1925 (2011).

12. Formolo, C. A. et al. Secretome signature of invasive glioblastoma multiforme. J. Proteome Res. 10, 3149-3159 (2011).

13. Tarassishin, L., Lim, J., Weatherly, D. B., Angeletti, R. H. \& Lee, S. C. Interleukin-1induced changes in the glioblastoma secretome suggest its role in tumor progression. J. Proteom. 99, 152-168 (2014).

14. Brandt, R., Hundelt, M. \& Shahani, N. Tau alteration and neuronal degeneration in tauopathies: mechanisms and models. BBA Mol. Basis Dis. 1739, 331-354 (2005).

15. Drechsel, D. N., Hyman, A., Cobb, M. H. \& Kirschner, M. Modulation of the dynamic instability of tubulin assembly by the microtubule-associated protein tau. Mol. Biol. Cell 3, 1141-1154 (1992).

16. Coussens, L. M. \& Werb, Z. Inflammation and cancer. Nature 420, 860-867 (2002).

17. Minelli, A., Bellezza, I., Conte, C. \& Culig, Z. Oxidative stress-related aging: a role for prostate cancer? BBA Rev. Cancer 1795, 83-91 (2009).

18. Leyns, C. E. \& Holtzman, D. M. Glial contributions to neurodegeneration in tauopathies. Mol. Neurodegener. 12, 50 (2017).

19. Tak, H. et al. Bimolecular fluorescence complementation; lighting-up tau-tau interaction in living cells. PLOS ONE 8, e81682 (2013).
20. Kim, S. et al. Functional interaction of neuronal Cav1. 3 L-type calcium channel with ryanodine receptor type 2 in the rat hippocampus. J. Biol. Chem. 282, 32877-32889 (2007)

21. Er, J. C. et al. NeuO: a fluorescent chemical probe for live neuron labeling. Angew. Chem. Int. Ed. 54, 2442-2446 (2015)

22. Lewis, J. et al. Neurofibrillary tangles, amyotrophy and progressive motor disturbance in mice expressing mutant (P301L) tau protein. Nat. Genet. 25, 402-405 (2000).

23. Paxinos, G. \& Franklin, K. B. The mouse brain in stereotaxic coordinates. (Elsevier Academic Press, Amsterdam, The Netherlands, 2008).

24. Forman, M. S. et al. Signature tau neuropathology in gray and white matter of corticobasal degeneration. Am. J. Pathol. 160, 2045-2053 (2002).

25. Lim, S. et al. Monitoring of intracellular tau aggregation regulated by OGA OGT inhibitors. Int J. Mol. Sci. 16, 20212-20224 (2015).

26. Kim, D. et al. Identification of disulfide cross-linked tau dimer responsible for tau propagation. Sci. Rep. 5, 15231 (2015).

27. Yeom, J., Kabir, M. H. \& Lee, C. Impact of data-dependent exclusion list based mass spectrometry on label-free proteomic quantification. Rapid Commun. Mass Spectrom. 29, 128-134 (2015).

28. Aruffo, A., Stamenkovic, l., Melnick, M., Underhill, C. B. \& Seed, B. CD44 is the principal cell surface receptor for hyaluronate. Cell 61, 1303-1313 (1990).

29. Miletti-González, K. E. et al. The CD44 receptor interacts with P-glycoprotein to promote cell migration and invasion in cancer. Cancer Res. 65, 6660-6667 (2005).

30. Nagano, O. \& Saya, H. Mechanism and biological significance of CD44 cleavage. Cancer Sci. 95, 930-935 (2004).

31. Okamoto, I. et al. Proteolytic cleavage of the CD44 adhesion molecule in multiple human tumors. Am. J. Pathol. 160, 441-447 (2002).

32. $\mathrm{Ku}, \mathrm{B} . \mathrm{M}$. et al. CHI3L1 (YKL-40) is expressed in human gliomas and regulates the invasion, growth and survival of glioma cells. Int. J. Cancer 128, 1316-1326 (2011).

33. Imperato, J. P., Paleologos, N. A. \& Vick, N. A. Effects of treatment on long-term survivors with malignant astrocytomas. Ann. Neurol. 28, 818-822 (1990).

34. Coyle, J. T. \& Puttfarcken, P. Oxidative stress, glutamate, and neurodegenerative disorders. Science 262, 689-689 (1993).

35. Lee, S.-G. et al. Oncogene AEG-1 promotes glioma-induced neurodegeneration by increasing glutamate excitotoxicity. Cancer Res. 71, 6514-6523 (2011).

36. Jain, R. K. Martin, J. D. \& Stylianopoulos, T. The role of mechanical forces in tumor growth and therapy. Ann. Rev. Biomed. Eng. 16, 321-346 (2014).

37. Goriely, A. et al. Mechanics of the brain: perspectives, challenges, and opportunities. Biomech. Model. Mechanobiol. 14, 931-965 (2015). 